\title{
MULTI TISSUE MODELLING OF DIFFUSION MRI SIGNAL REVEALS VOLUME FRACTION BIAS
}

\author{
Matteo Frigo ${ }^{1 *}$, Rutger H. J. Fick ${ }^{2 *}$, Mauro Zucchelli ${ }^{1}$, Samuel Deslauriers-Gauthier ${ }^{1}$, Rachid Deriche ${ }^{1}$ \\ ${ }^{1}$ Athena Team, Inria Sophia-Antipolis Méditerranée, Université Côte D’Azur, France \\ ${ }^{2}$ Therapanacea, France \\ * These authors contributed equally to this work.
}

\begin{abstract}
This paper highlights a systematic bias in white matter tissue microstructure modelling via diffusion MRI that is due to the common, yet inaccurate, assumption that all brain tissues have a similar $T_{2}$ response. We show that the concept of "signal fraction" is more appropriate to describe what have always been referred to as "volume fraction". This dichotomy is described from the theoretical point of view by analysing the mathematical formulation of the diffusion MRI signal. We propose a generalized multi tissue modelling framework that allows to compute the actual volume fractions. The Dmipy implementation of this framework is then used to verify the presence of this bias in four classical tissue microstructure models computed on two subjects from the Human Connectome Project database. The proposed paradigm shift exposes the research field of brain tissue microstructure estimation to the necessity of a systematic review of the results obtained in the past that takes into account the difference between the concept of volume fraction and tissue fraction.
\end{abstract}

Index Terms- Diffusion MRI, White Matter, Microstructure, Generalized Multi Tissue Modelling

\section{INTRODUCTION}

Diffusion MRI (dMRI) is able to probe brain tissue microstructure in-vivo non-invasively. Two of the most commonly studied microstructural features are the intra-axonal and cerebro-spinal fluid (CSF) volume fractions and several microstructural models have been proposed to retrieve them $[1,2,3]$. These models differ in the type of signal model used to represent a given tissue type (e.g. diffusion within a stick or cylinder for modeling the intra-axonal diffusion)

This work was funded by the European Research Council (ERC) under the European Union's Horizon 2020 research and innovation program (ERC Advanced Grant agreement No 694665: CoBCoM - Computational Brain Connectivity Mapping). Data were provided by the Human Connectome Project, WU-Minn Consortium (Principal Investigators: David Van Essen and Kamil Ugurbil; 1U54MH091657) funded by the 16 NIH Institutes and Centers that support the NIH Blueprint for Neuroscience Research; and by the McDonnell Center for Systems Neuroscience at Washington University. RHJF is employed by Therapanacea. or their particular assumptions on the model parameters (e.g. fixed parallel diffusivity, tortuosity, etc.). To our knowledge, all these models are based on the normalised diffusion signal and thus assume that the signal fractions of the different compartments sum to one. However, the concepts of signal fraction and tissue volume fraction are not interchangeable. The first measures the linear relation between the signal generated by a single tissue compartment and the acquired signal, while the second measures the volume of single tissue compartment that is present in the voxel. To understand the origin of this difference, consider that given the proton density $[H]$, the repetition time $T R$, the echo time $T E$ and the $T_{1}$ and $T_{2}$ times of a specific tissue, the resulting signal intensity in a spin echo sequence is given by $S \sim[H] \cdot\left(1-e^{-T R / T_{1}}\right) \cdot e^{T E / T_{2}}$. Since the $T_{2}$ time of the white matter (WM) is higher than the one of the CSF, the resulting signal amplitude will be higher in regions populated only by CSF. A simple normalization of the dMRI signal by the non-diffusion-weighted signal $S_{0}$ does not take into account these differences and is equivalent to assuming that each tissue has the same $S_{0}$ response, which is not true in general (see [4] and Table 1). Acquiring images with multiple TE [5] would still not solve this issue, as this technique allows to estimate the composite $T_{2}$ time in each voxel, but the $T_{2}$ response of the single tissues would still be unknown.

All the multi-compartment (MC) models of tissue microstructure that aim at estimating the volume fraction of tissues are actually describing the signal fraction. A possible solution to a similar problem has been proposed by Jeurissen et al. [6] in the context of fiber Orientation Distribution Function (fODF) estimation for multi-shell dataset, where the modelled signal is not normalised by the signal amplitude. In this work, we propose a technique inspired by the one presented in [6] that allows the estimation of the true tissue volume fraction in standard MC models and Spherical Mean (SM) MC models. The resulting framework will open the possibility to analyse unbiased tissue volume fractions estimated from dMRI signals in heterogeneous tissues.

The recently proposed Dmipy toolbox [7] provides an implementation of this generalized tissue modelling (GTM) and 
was employed to show the impact of GMT on two formulations of the widely studied multi-compartment model Neurite Orientation Dispersion and Density Imaging (NODDI) [1]. Our results on two subjects from the Human Connectome Project (HCP) database show that, as previously discussed, the concepts of signal fraction and volume fraction are not interchangeable. Moreover, all the studies involving white matter tissue microstructure models should be reinterpreted taking into account the difference between signal fraction and volume fraction.

\section{THEORY}

The Pulsed Gradient Spin Echo (PGSE) signal measured by $\mathrm{dMRI}$ can be represented as the product of amplitude $S_{0}$ (nondiffusion-weighted) and shape $E$ (diffusion-weighted):

$$
S(G, n, \Delta, \delta, T E)=S_{0}(T E) \cdot E(G, n, \Delta, \delta)
$$

where $T E[s]$ is the echo time, $G[T / m]$ is the gradient strength, $n$ is the gradient orientation, $\Delta[s]$ is the pulse separation and $\delta[s]$ is the pulse duration. From equation (1) we can notice that the signal amplitude $S_{0}$ depends only on TE, while the signal shape $E$ depends on all the other acquisition parameters, which will be denoted as $A=[G, n, \Delta, \delta]$. To our knowledge, all multi-compartment (MC) models, among which we mention NODDI [1], SMT [2] and ActiveAx [3], aim at fitting the signal shape $E$ as the ratio of the PGSE signal $S$ and the $S_{0}$ amplitude. The general formulation of all these MC models is

$$
E(A)=\frac{S(A, T E)}{S_{0}(T E)}=\sum_{i=1}^{N_{c}} \phi_{i} \cdot E_{i}\left(A, \mathbf{p}_{i}\right)
$$

where $\phi_{i}$ is the volume fraction of compartment $i, N_{c}$ is the number of considered compartments and $\mathbf{p}_{i}$ is the parameter vector for the model of compartment $i$. The implicit assumption lying behind this formulation is that all the modelled tissues have similar $T_{2}$ relaxation times, which is not generally true. Table 1 shows how this difference in $T_{2}$ times is reflected in the $S_{0}$ response of three different tissues. In order to take into account the different $T_{2}$ times of each tissue, we define the Generalised Multi-Tissue Multi-Compartment (MT-MC) model as follows:

$$
S(A, T E)=\sum_{i=1}^{N_{c}} f_{i} \cdot S_{0}^{i}(T E) \cdot E_{i}\left(A, \mathbf{p}_{i}\right)
$$

where $f_{i}$ is the volume fraction of compartment $i$ and $S_{0}^{i}(T E)$ is the $S_{0}$ response of the tissue modelled by compartment $i$. Notice that $\phi_{i}$ and $f_{i}$ are not equivalent in general, as the former describes the signal fraction of compartment $i$ and the latter describes the volume fraction of the tissue modelled by compartment $i$. The only case in which they will be equivalent is when all the tissues described by the MC model have equivalent $S_{0}$ responses. For this reason we say that $\phi_{i}$ is a biased estimator of $f_{i}$.

The same argument that led to (3) can be applied to the case of the SM representation of dMRI signals inspired by the Spherical Mean Technique (SMT) of Kaden et al. [2]. The SM representation describes the spherical mean of the signal in each $b$-shell instead of the dMRI signal in each acquired direction. Integrating both sides of equation (3) we obtain an orientation-invariant representation of the dMRI signal which reads as follows

$$
\begin{aligned}
\bar{S}_{b} & =\int_{S^{2}} S_{b}(n) d n=\sum_{i=1}^{N_{c}} f_{i} \cdot S_{0}^{i} \cdot \int_{S^{2}} E_{i}^{b}\left(n, \mathbf{p}_{i}\right) d n \\
& =\sum_{i=1}^{N_{c}} f_{i} \cdot S_{0}^{i} \cdot \bar{E}_{i}^{b}\left(\mathbf{p}_{i}\right)
\end{aligned}
$$

where $b$ is the b-value of the shell whose signal is being modelled, $\bar{S}_{b}$ is the spherical mean of the signal on shell $b$ and the dependence on $T E$ has been omitted for the sake of readability. We will refer to equation (4) as the Multi-Tissue SM (MT-SM) representation of a microstructure model.

\section{METHODS}

The fitting of a MT-MC or a MT-SM model is designed as a 2-steps process. In the first step an optimization problem is defined to retrieve the possibly non-linear parameters $\mathbf{p}_{i}$ that determine each compartment model and the signal fractions $\phi_{i}$. The optimisation problem corresponding to the forward model in equation (3) reads as follows

$$
\phi_{i}^{*}, \mathbf{p}_{i}^{*}=\underset{\phi_{i}, \mathbf{p}_{\mathbf{i}}}{\arg \min }\left\|\frac{\hat{S}}{S_{0}}-\sum_{i=1}^{N_{c}} \phi_{i} E_{i}\left(\mathbf{p}_{i}\right)\right\|_{2}^{2}
$$

where $\hat{S}$ is the acquired dMRI signal and the dependence on the acquisition parameters has been omitted for the sake of readability. The SM case is analogous. The retrieved parameters $\mathbf{p}_{i}^{*}$ are fixed to the ones estimated in the first step and a second optimization is performed in order to estimate the volume fractions of each compartment as follows

$$
f^{*}=\underset{f_{i}}{\arg \min }\left\|\hat{S}-\sum_{i=1}^{N_{c}} f_{i} \cdot S_{0}^{i} \cdot E_{i}\left(\mathbf{p}_{i}^{*}\right)\right\|_{2}^{2}
$$

where equation (6) solves the volume fraction retrieval problem for MT-MC models and the problem is solved analogously for the MT-SM model. Notice that the latter is a convex linear least squares problem since the non linearity of $E_{i}$ with respect of $\mathbf{p}$ has been solved by fixing the parameters $\mathbf{p}_{i}^{*}$.

The first step is actually the same that would be employed in the estimation of a MC model of the form (2) that does not include MT modelling. Performing this fitting allows to 


\begin{tabular}{c|ccccccccc} 
Subject & 105923 & 106521 & 108323 & 109123 & 113922 & 116726 & 133019 & 140117 & 156334 \\
\hline$S_{0}^{W M}$ & $2.26 \mathrm{e}+03$ & $2.22 \mathrm{e}+03$ & $1.22 \mathrm{e}+03$ & $1.07 \mathrm{e}+03$ & $1.25 \mathrm{e}+03$ & $2.18 \mathrm{e}+03$ & $1.98 \mathrm{e}+03$ & $2.37 \mathrm{e}+03$ & $1.93 \mathrm{e}+03$ \\
$S_{0}^{C S F}$ & $1.32 \mathrm{e}+04$ & $9.15 \mathrm{e}+03$ & $8.47 \mathrm{e}+03$ & $1.19 \mathrm{e}+04$ & $1.12 \mathrm{e}+04$ & $1.27 \mathrm{e}+04$ & $8.68 \mathrm{e}+03$ & $9.69 \mathrm{e}+03$ & $1.00 \mathrm{e}+04$
\end{tabular}

Table 1. $S_{0}$ response of WM and CSF for nine HCP [8] subjects obtained via the heuristic approach of Dhollander et al. [9] implemented in Dmipy [7]. Notice that $S_{0}^{W M}<S_{0}^{C S F}$. These results are in line with the ones reported in [6, 7].

achieve a correct estimation of the compartment-specific parameters $\mathbf{p}_{i}$, but the fitted signal fractions $\phi_{i}^{*}$ will be biased estimators of $f_{i}^{*}$, as stated in Section 2. The second step is a linear constrained minimization problem that can be solved with dedicated routines [10]. Since the single tissue-response estimations $S_{0}^{i}$ may vary over the analysed volume, we avoid enforcing unitary sum of the volume fractions. Other reasons are thoroughly discussed in [11]. Splitting the optimization in two steps answers to the necessity to have both the best possible estimation of the model-specific parameters $\mathbf{p}_{i}$ and an accurate approximation of the volume fractions. The procedure is designed in such a way that the first step is focused on the approximation of the signal shape while the second gives the volume fractions by modulating only the signal amplitude.

\section{RESULTS}

The studied dataset is composed of nine randomly chosen subjects from the HCP database preprocessed with the minimal preprocessing pipeline [8]. For each subject we computed the signal fraction and the volume fraction for the WM and the CSF compartment via NODDI and its spherical mean formulation SM-NODDI. A white matter mask was obtained from the subcortical segmentation of Freesurfer [12]. The estimation of the $S_{0}^{i}$ responses for the WM and CSF tissues was performed using the heuristic approach of Dhollander et al. [9]. Figure 1 shows the comparison between the average distribution across the nine subjects of the re-normalised WM signal fraction and normalised WM volume fraction for each studied model. The graphs highlight the quantitative difference

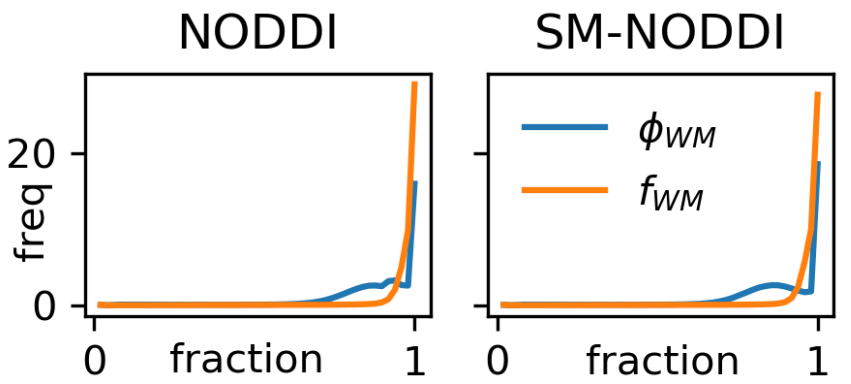

Fig. 1. Average distribution of the signal fraction $\phi_{W M}$ (blue) and the volume fraction $f_{W M}$ (orange) of the WM compartment obtained on the studied subjects. The variance is $<5 \%$ and would not be visible in the plot.
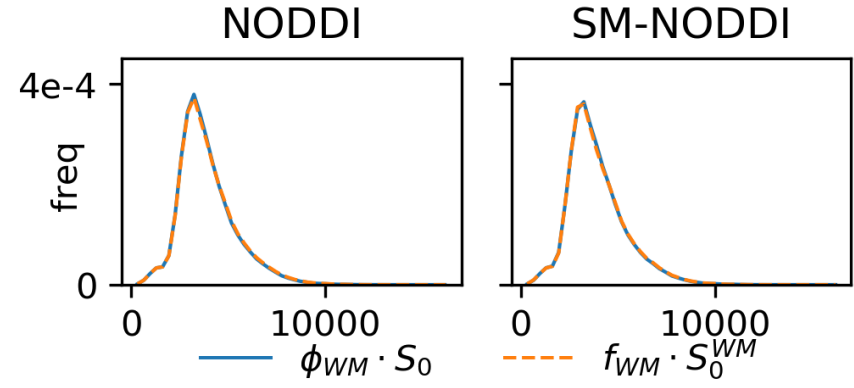

Fig. 2. Distribution of $\phi_{W M} \cdot S_{0}$ in blue and $f_{W M} \cdot S_{0}^{W M}$ in orange for each considered technique in subject 105923. An analogous behaviour appears in every other studied subject.

between signal fractions and volume fractions, giving empirical evidence to the fact that $\phi_{i}$ is a biased estimator of $f_{i}$. Moreover, the WM volume fraction results to be closer to 1 than the signal fraction. This is coherent with the fact that $S_{0}^{W M} \leq S_{0}$ in healthy brains, as the product $f_{W M} \cdot S_{0}^{W M}$ in equation (3) should be directly proportional to the product $\phi_{W M} \cdot S_{0}$ that can be deduced from equation (2). This fact is exemplified in Figure 2, where a direct comparison of the distribution of the two products is presented for the two techniques. Figure 3 shows the normalised WM signal fraction $\phi_{W M}$ and the normalised WM volume fraction $f_{W M}$ computed with SM-NODDI and SM-Activax. The remarkable differences between the signal fractions in the two hemispheres are not present in the volume fractions. Moreover, the WM volume fraction does not present the scattered artifacts that characterize the value of $\phi_{W M}$ in both hemispheres.

\section{DISCUSSION AND CONCLUSIONS}

This study highlights a bias that is present in all multicompartment models that describe multiple tissues at the same time. The fact that the reported results uniquely involve equivalent formulations of NODDI shouldn't be read as an analysis of the model per se. On the contrary, it is presented as an empirical example of the conceptual difference between the signal fraction and the volume fraction. Our preliminary results highlight a remarkable difference between these two. Changing the focus of the studies from signal fraction to volume fraction represents a major paradigm shift in white matter modelling. This translates into the necessity for a radical reinterpretation of the studies based on microstructure models that simultaneously describe multiple tissues having 


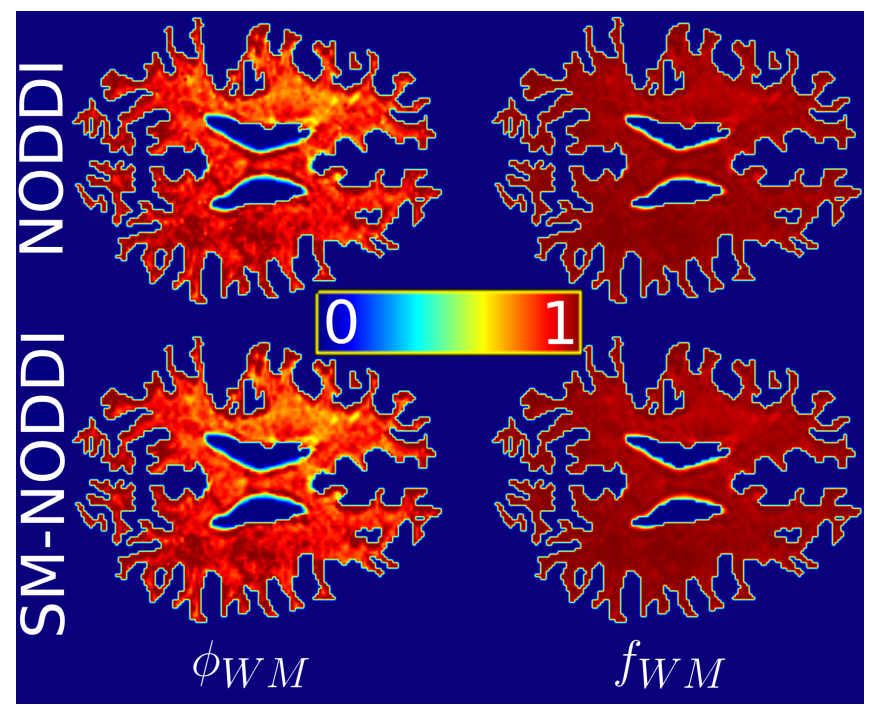

Fig. 3. Normalised signal fraction $\phi_{W M}$ and normalised volume fraction $f_{W M}$ of WM tissue compartment computed with NODDI and SM-NODDI on subject 105923. Warmer colors (red) indicate higher values of the indicated fraction.

different $S_{0}$ responses.

In our experiments we used the heuristic approach of Dhollander et al. [9] to compute the single tissue responses. Nevertheless, the proposed framework will be well defined also for other single tissue responses estimation algorithms. A possible alternative is the $T_{1}$-segmentation-based technique of Jeurissen et al. [6].

The results presented in Figure 2 suggest that, under the assumption that the signal shape of each compartment is linearly independent, it is possible to recover the volume fractions by means of a simple rescaling of the signal fractions. This possibility will be explored in future works.

The bias that we highlighted both from the theoretical and empirical point of view is a relevant issue in white matter microstructure and we advocate the use of generalized multi tissue modelling to re-establish the meaningfulness of the concept of tissue volume fraction. More studies will be needed to investigate the effects of GTM in both healty and non-healty subjects.

\section{REFERENCES}

[1] Hui Zhang, Torben Schneider, Claudia A WheelerKingshott, and Daniel C Alexander, "Noddi: practical in vivo neurite orientation dispersion and density imaging of the human brain," Neuroimage, vol. 61, no. 4, pp. 1000-1016, 2012.

[2] Enrico Kaden, Frithjof Kruggel, and Daniel C Alexander, "Quantitative mapping of the per-axon diffusion coefficients in brain white matter," Magnetic resonance in medicine, vol. 75, no. 4, pp. 1752-1763, 2016.

[3] Daniel C Alexander, Penny L Hubbard, Matt G Hall, Elizabeth A Moore, Maurice Ptito, Geoff JM Parker, and Tim B Dyrby, "Orientationally invariant indices of axon diameter and density from diffusion mri," Neuroimage, vol. 52, no. 4, pp. 1374-1389, 2010.

[4] M Just and M Thelen, "Tissue characterization with $\mathrm{t} 1, \mathrm{t} 2$, and proton density values: results in 160 patients with brain tumors.," Radiology, vol. 169, no. 3, pp. 779$785,1988$.

[5] Jelle Veraart, Dmitry S. Novikov, and Els Fieremans, "Te dependent diffusion imaging (teddi) distinguishes between compartmental $\mathrm{t} 2$ relaxation times," NeuroImage, vol. 182, pp. 360 - 369, 2018.

[6] Ben Jeurissen, Jacques-Donald Tournier, Thijs Dhollander, Alan Connelly, and Jan Sijbers, "Multi-tissue constrained spherical deconvolution for improved analysis of multi-shell diffusion mri data," NeuroImage, vol. 103, pp. 411-426, 2014.

[7] Rutger H. J. Fick, Demian Wassermann, and Rachid Deriche, "The dmipy toolbox: Diffusion mri multicompartment modeling and microstructure recovery made easy," Frontiers in Neuroinformatics, vol. 13, no. 64, 2019.

[8] Matthew F Glasser, Stamatios N Sotiropoulos, J Anthony Wilson, Timothy S Coalson, Bruce Fischl, Jesper L Andersson, Junqian Xu, Saad Jbabdi, Matthew Webster, Jonathan R Polimeni, et al., "The minimal preprocessing pipelines for the human connectome project," Neuroimage, vol. 80, pp. 105-124, 2013.

[9] Thijs Dhollander and Alan Connelly, "A novel iterative approach to reap the benefits of multi-tissue csd from just single-shell $(+\mathrm{b}=0)$ diffusion mri data," in Proc ISMRM, 2016, vol. 24, p. 3010.

[10] Michael JD Powell, "A view of algorithms for optimization without derivatives," Mathematics Today-Bulletin of the Institute of Mathematics and its Applications, vol. 43, no. 5, pp. 170-174, 2007.

[11] Flavio Dell'Acqua and J-Donald Tournier, "Modelling white matter with spherical deconvolution: How and why?," NMR in Biomedicine, vol. 32, no. 4, pp. e3945, 2019.

[12] Bruce Fischl, David H Salat, Evelina Busa, Marilyn Albert, Megan Dieterich, Christian Haselgrove, Andre Van Der Kouwe, Ron Killiany, David Kennedy, Shuna Klaveness, et al., "Whole brain segmentation: automated labeling of neuroanatomical structures in the human brain," Neuron, vol. 33, no. 3, pp. 341-355, 2002. 